IdeAs

Idées d'Amériques

18 | 2021

Frontières dans les Amériques - Intégration, sécurité

et migrations

\title{
Bicentenaire au Guatemala : mythe national et (in)dépendances
}

\section{Clara Duterme}

\section{(2) OpenEdition \\ Journals}

Édition électronique

URL : https://journals.openedition.org/ideas/11488

DOI : $10.4000 /$ ideas. 11488

ISSN : 1950-5701

Éditeur

Institut des Amériques

Référence électronique

Clara Duterme, «Bicentenaire au Guatemala : mythe national et (in)dépendances », IdeAs [En ligne],

18 | 2021, mis en ligne le 01 octobre 2021, consulté le 21 octobre 2021. URL : http://

journals.openedition.org/ideas/11488; DOI : https://doi.org/10.4000/ideas.11488

Ce document a été généré automatiquement le 21 octobre 2021.

\section{cc) (1)}

IdeAs - Idées d'Amériques est mis à disposition selon les termes de la licence Creative Commons

Attribution - Pas d'Utilisation Commerciale - Pas de Modification 4.0 International. 


\title{
Bicentenaire au Guatemala : mythe national et (in)dépendances
}

\author{
Clara Duterme
}

1 Le 27 février 2021, sur le site archéologique maya de Iximché, le président de la république guatémaltèque Alejandro Giammetti assistait à la cérémonie d'ouverture des célébrations du Bicentenaire. À l'occasion de l'anniversaire de la signature de l'acte d'Indépendance, des évènements sportifs et culturels ont été prévus dans les différents départements du pays jusqu'au 15 septembre 2021, jour de la fête nationale. L'unité nationale constitue le socle des discours et de la mise en scène mobilisant les symboles nationaux : l'hymne joué par l'orchestre national, le territoire incarné par une torche nommée « feu patriotique » (fuego patrio), destinée à passer par chaque municipe du pays pour allumer la flamme nationale, le choix même du lieu qui puise dans les références au passé antique maya. Le Bicentenaire est décrit comme un moment historique visant à célébrer « les traditions, la gastronomie, les héros de différentes époques et l'essence de la Nation », « unidos en la celebración [y] el rescate de la identidad nacional, reconociendo nuestra diversidad, buscando la reconciliacion y la armonia social $»^{1}$.

2 Si les mots et les symboles mobilisés s'inscrivent dans une célébration classique du mythe national, ces déclarations sonnent remarquablement creuses au regard du contexte social et politique qui a marqué l'année du Bicentenaire pour le Guatemala. La commémoration du Bicentenaire, organisée par l'État, le ministère des Sports et l'Institut guatémaltèque du Tourisme, a été l'occasion pour les observateurs de rappeler le constat alarmant de la situation sociale du pays : le Guatemala continue d'être l'un des pays les plus dangereux et les plus inégaux au monde, une inégalité qui s'est même aggravée ces dernières années - l'indice Gini étant passé de 0,54 en 2002 à 0,48 en 2014 - et qui touche en particulier les populations indigènes, lesquelles constituent plus de la moitié de la population. L'analphabétisme et la malnutrition enfantine se classent parmi les plus élevés au monde. L'incertitude économique a été aggravée par la crise du Coronavirus, qui a fait s'effondrer les ressources du tourisme international - la seconde source de devises du pays -, tandis que la gestion de la crise sanitaire par le gouvernement a été fortement critiquée. Des soupçons de 
malversations financières ont encore accentué un mécontentement social exacerbé visà-vis de la corruption généralisée (Sieder R., 2001, Heasley N., et al. 2001), entraînant un mouvement de protestation exigeant la démission du président.

Dans ce climat social tendu, l'anniversaire des 200 ans d'existence du pays en tant qu'entité souveraine et indépendante interroge les enjeux actuels des relations de la population avec l'État, ainsi que le sentiment d'appartenance nationale, et ce dans un contexte notoirement complexe: dans un pays historiquement, ethniquement et socialement divisé, aux institutions imprégnées de racisme systémique (Hale, C. 2006), où, 25 ans après la signature des Accords de paix ${ }^{2}$, une grande partie de la population continue de faire l'expérience d'un continuum de violence (Bourgois P., 2012), politique notamment, et où le travail d'adhésion à la « fiction nationale » (Thiesse A.-M., 2010) reste encore largement à faire.

\section{Le mythe désenchanté de l'unité nationale}

4 La construction du sentiment national repose en partie sur l'invention, ou la mise en récit, d'un passé commun. Or le Guatemala entretient avec son passé une relation ambivalente, qu'il s'agisse de l'époque de l'Indépendance nationale ou de l'époque plus proche du conflit armé, qui a joué un rôle central dans la refondation de la Nation. La célébration du Bicentenaire a suscité un foisonnement de réflexions historiographiques au sein du monde académique, portant un regard critique sur l'enseignement de l'histoire et sur la construction du mythe national. L'Université publique de San Carlos a ainsi mis en place une "commission pour une commémoration (et non une célébration) critique du Bicentenaire ", présidée par l'historien Mario Roberto Morales. Avec une approche critique, qui met l'accent sur l'histoire sociale et populaire, s'intéressant aux acteurs sociaux exclus du mythe national comme de l'historiographie, en particulier celle des populations indigènes qui n'ont jamais été pleinement intégrées dans la communauté nationale. Depuis la publication en 1998 du fameux ouvrage de Severo Martínez Peláez La Patria del criollo, l'illusion historique d'une unité nationale a fait l'objet de nombreux débats. Le projet indépendantiste ne fut pas le résultat d'un projet politique, mais d'abord d'une révolution libérale, menée par les élites criollas, pour leur propre bénéfice. L'indépendance n'a en rien bouleversé les inégalités sociales héritées de la période coloniale et articulées à une stratification ethnique. Les criollos descendants d'Espagnols, considérés comme blancs - détenaient le pouvoir politique et économique. À l'autre bout du spectre, les Indiens (indios) constituaient la classe laborieuse, ayant un accès très restreint à la propriété de la terre, tandis qu'entre les deux se répartissait le reste de la société en diverses catégories métisses.

5 Le Guatemala est le plus souvent décrit comme une nation comportant trois groupes ethniques principaux: les Indiens (dénommés aujourd'hui «indígenas », après les transformations des années 1970 et post-conflit), la minorité noire Xinca et les "ladinos", terme bancal qui englobe "tous les autres", souvent définis de façon simplifiée par le terme «non-indiens». Si les populations indigènes ont été historiquement exclues de la communauté nationale, cet état de fait a été remis en question à partir des années 1980-1990.

6 Le conflit armé, puis les Accords de paix négociés entre 1994 et 1996, ont constitué un moment charnière dans l'histoire de la reconnaissance des droits des peuples indigènes guatémaltèques. En effet, la violence politique du conflit armé, qui opposait l'armée 
(soutenant un gouvernement dictatorial) et des mouvements de guérilla marxistes, ne peut être abordée sans considérer le contexte ethnique dans lequel elle s'est déployée. La figure de l'Indien (indio) a historiquement été associée à la crainte du soulèvement par les élites «blanches». On ne peut ignorer les liens entre cette perception ethnique et le développement de tactiques militaires de terreur, qui ont ciblé des populations indigènes intrinsèquement considérées comme subversives (Rodriguez Maeso, 2010). L'intensité des violences génocidaires a entraîné l'intervention de la communauté internationale pour imposer la paix, ainsi que l'introduction d'une «culture de la démocratie " qui s'accordait à la politique étrangère des États-Unis de l'époque (Couffignal G., 1992). L'injonction à la reconnaissance culturelle des peuples autochtones qui s'était imposée sur la scène internationale, portée par le courant indianiste en Amérique depuis les années 1960 et 1970 (Lavaud J.-P., \& F. Lestage, 2006), a également influé sur les négociations de paix. Bien que la Constitution guatémaltèque ne reconnaisse pas officiellement la nation comme pluriethnique et pluriculturelle, les enjeux du multiculturalisme ont partiellement été intégrés à la législation nationale, qui garantit un certain nombre de droits culturels, tels que l'éduction bilingue ou la non-discrimination. Cependant, l'application de ces textes de loi n'a jamais été réellement appuyée par l'État.

7 C'est dans le contexte de post-conflit des années 1990 que les références aux droits des indigènes et aux droits humains se sont développées au Guatemala, dans un contexte marqué par le concept de justice transitionnelle et l'idée d'une "réconciliation " nationale, dont la réalité est contestable à double titre : d'abord, parce que les outils de réconciliation mis en place essentiellement par les acteurs de la communauté internationale et de l'Église n'ont jamais été pleinement pris en main par l'État et, d'autre part, parce que le terme suppose un état d'harmonie préalable qui n'a jamais existé dans l'histoire du pays (Manz B., 2008). Le processus de reconnaissance des victimes a ainsi constitué la première étape pour, non pas restituer, mais bien produire une citoyenneté dont les populations indigènes avaient toujours été exclues (Rodríguez Maeso S., 2010). Les défenseurs des droits humains ont engagé depuis bientôt 25 ans un travail de mémoire qui s'appuie essentiellement sur la reconnaissance des victimes au travers du prisme de la justice transitionnelle et de la condamnation des crimes contre l'humanité. Or, aujourd'hui encore, il n'existe pas réellement de récit commun sur le passé violent guatémaltèque ni de consensus sur le type de justice garantie par un État de droit ${ }^{3}$. La perception des violences du conflit reste un point de rupture politique majeur au sein du pays, entre une partie de la classe politique soutenant que les militaires ont agi dans l'intérêt du pays, pour protéger la population de la subversion, tandis que les défenseurs des «droits humains» (un terme fréquemment utilisé de façon péjorative par leurs opposants), soutenus par la communauté internationale, dénoncent la violence génocidaire exercée à l'encontre de la population.

\section{Dépendances et défaillances de l'État}

8 Si les divisons historiques et sociales remettent en question l'idée d'un sentiment partagé d'appartenance nationale au Guatemala, la relation actuelle à l'État est également problématique. Bien que les Accords aient tenté de poser les nouvelles bases d'un système démocratique, à travers des réformes constitutionnelles et institutionnelles, force est de constater aujourd'hui que l'État guatémaltèque constitue 
un État « en faillite ». Cette faiblesse de l'État peut s'expliquer par l'absence quasi-totale de recettes fiscales, mais également par un système électoral fortement clientéliste et instable. Le constat fait par Willibald Sonnleitner (2009), selon lequel « [en] l'absence d'une participation active de la population, la démocratisation guatémaltèque se caractérise par une classe politique singulièrement "personnaliste" et "court-termiste", ainsi que par un système de partis désagrégé à l'extrême", conserve toute sa pertinence, alors que plus de la moitié des partis représentés pour l'élection présidentielle de 2019 avaient été fondés moins de deux ans avant l'élection.

9 Il faut souligner que l'accès des habitants - en particulier des populations indigènes aux institutions reste extrêmement limité, en raison d'un éloignement tant géographique (lié à la centralisation du pouvoir dans la capitale) que social. La barrière linguistique et le racisme systémique ancrés dans les institutions étatiques (Hale C., 2006) tendent à exclure symboliquement les Indiens. Aux yeux d'une grande partie des Guatémaltèques ces institutions, chroniquement sous-dotées en personnel et en moyens, ne font rien pour eux, ce qui provoque un sentiment de disjonction entre la lettre de la loi, qui garantit l'égalité et l'accès aux droits, et la réalité vécue. Les réponses apportées localement vont conduire à compenser les manques par des initiatives privées, communautaires ${ }^{4}$, ou en se tournant vers l'aide de la communauté internationale, présente à une telle échelle que l'on peut parler d'«ONGisation » de la sphère publique (Vanthuyne K., 2014) au Guatemala.

10 La confiance dans les dirigeants politiques est encore affaiblie par les nombreuses affaires de corruption qui touchent les plus hauts niveaux de l'État. La colère sociale qui couvait depuis la dissolution de la Commission internationale contre l'impunité guatémaltèque (CICIG) en 2019 s'est ré-enflammée suite à la destitution, en juillet 2021, du chef de la Fiscalía Especial contra la Impunidad (Bureau du ministère public contre l'impunité), Juan Francisco Sandovar, ce qui entraîna des manifestations dans tout le pays demandant la démission du président, soupçonné d'être à l'origine de l'évincement d'un fonctionnaire à la tête d'enquêtes qui risquaient de révéler les malversations de son gouvernement.

11 Cette affaire s'inscrit dans la continuité de nombreux scandales de corruption ayant touché les gouvernements successifs et, surtout, elle fait suite à l'éviction de la CICIG. Bien qu'il s'agisse d'un organisme indépendant destiné à soutenir l'État guatémaltèque dans sa lutte contre la corruption, cette commission a pourtant été démantelée en 2019, après plusieurs affaires d'envergure ayant impliqué des représentants du gouvernement $^{5}$. Le travail mené par la CICIG étant considéré par beaucoup comme l'un des seuls leviers efficaces contre la corruption généralisée dans le pays, l'arrêt de ses activités avait provoqué une indignation que l'éviction de Sandoval (lui-même membre de la commission avant de continuer son action auprès de la Fiscalía) a ranimé. Dès le 29 juillet, des grèves et des manifestations sont organisées dans le pays, qui n'est pourtant pas coutumier des grandes mobilisations sociales, pour dénoncer les actes de corruption des institutions étatiques, une protestation qui se poursuivra sporadiquement au cours du mois d'août. Ces manifestations constituent un évènement dans un pays où les modalités d'actions sociales sont décrédibilisées par les autorités, à travers des discours qui renvoient toute mobilisation du côté de « la violence » (Benson P., 2004).

12 La proximité de la date du Bicentenaire a conduit les opposants à se positionner sur les enjeux d'appartenance nationale. Dans tout le pays, des marches ou des évènements 
contre le Bicentenaire ont été organisés, le plus souvent à l'initiative des comités paysans, autour des slogans : "Nada que celebrar " (il n'y a rien à célébrer) ou « No al Bicentenario ». À Quetzaltenango, la seconde ville du pays, les manifestants ont dénoncé leur statut de "Guatémaltèque[s] par décret et non par auto-identification », tandis que sur l'iconique arche d'entrée de la ville de Chichicastenango, une banderole annonçait : « 200 ans de racisme, persécutions, génocide, corruption, pillage, impunité ». Ces contre-célébrations marquent la mise en scène d'une distanciation formelle avec le mythe national et l'intégration du positionnement critique développé dans les milieux académiques par une partie de la population.

200 ans après la date officielle de fondation de l'État guatémaltèque, l'histoire nationale semble surtout le produit de ruptures successives ; l'unité et l'harmonie invoquées dans les discours commémoratifs sont loin d'être atteintes, alors que la réalité vécue par la majorité de la population semble surtout marquée par l'absence de réel socle de communauté nationale et le manque de confiance en l'État pour incarner le modèle démocratique promis au moment des Accords de paix et assumer les responsabilités qui lui échoient. Dans cette situation, le sentiment d'appartenance est à interroger dans le cadre des pratiques "bricolées" par les citoyens pour faire face aux défaillances de l'État, qui s'inscrivent dans des dynamiques mettant en relation les initiatives communautaires et les ressources internationales, l'État intervenant souvent comme un échelon intermédiaire nécessaire. Les évènements qui ont marqué localement la date du Bicentenaire s'inscrivent en faux par rapport aux célébrations formelles - qui ont culminé dans un défilé militaire le jour de la fête nationale. Dans la région Quiché, elles ont pris la forme de cérémonies mayas dédiées à la commémoration des morts de la colonisation en même temps que ceux de la pandémie, tandis que dans la région Ixcán, les représentants indigènes annonçaient célébrer «la résistance, pas le Bicentenaire ", tout en réclamant aux autorités nationales de prêter enfin attention à la partie oubliée de la population. Les exemples sont multiples de la façon dont les mouvements contestataires puisent dans l'histoire et l'iconographie de la résistance indigène face à la colonisation pour contester la réalité de l'unité nationale - tant dans sa perception que dans son application concrète - et revendiquer une expérience commune de l'indigénéité caractérisée par l'exclusion, qui se décline à l'échelle nationale ${ }^{6}$ et qui transcende les frontières du continent.

\section{BIBLIOGRAPHIE}

Benson, Peter, « Nothing to See Hear », Anthropological Quaterly, vol. 77, n³, 2004, p. 435-467.

Bourgois, Philippe « Théoriser la violence en Amérique. Retour sur trente ans d'ethnographie », L'Homme, n²03-204, 2012, p. 139-168.

Couffignal, Georges, «L'Amérique centrale 1979-1990 : de l'internationalisation à la régionalisation des crises locales ", Cultures\&Conflits, $n^{\circ} 8$, hiver 1992, mis en ligne le 27 janvier 2003, consulté le 02 octobre 2016 [URL : http://conflits.revues.org/523; DOI : 10.4000/conflits. 523]. 
Hale, Charles, Más que un indio (more than an Indian): Racial ambivalence and the paradoxe of Neoliberal Multiculturalism in Guatemala, Santa Fe, School for Advanced Research Press, 2006.

Heasley, Nathanael, Hurley, Rodger, Irwin, Kara E. et al., « Impunity in Guatemala: The State's failure to provide Justice in the Massacre Cases », American University International Law Review, 16 (5), 2001, p. 1115-1194.

Lavaud, Jean-Pierre et Françoise Lestage, « Les redéfinitions de l'indianité. Historique, réseaux, discours, effets pervers ", Esprit, (1), 2006, p. 42-64.

Manz, Béatrice, « The continuum of violence in Post-war Guatemala », Social Analysis, 52(2), 2008, p.151-164.

Martínez Peláez, Severo, La Patria del criollo: ensayo de interpretación de la realidad colonial guatemalteca, México, Fondo de Cultura Económica, 1998.

Rodríguez Maeso, Silvia, « Política del testimonio y reconocimiento en las comisiones de la verdad guatemalteca y peruana: En torno a la figura del «indio subversivo », Revista Crítica de Ciências Sociais, $\mathrm{n}^{\circ} 88,2010$, p. 23-55.

Sieder, Rachel, « Rethinking Citizanship, reforming the Law in Postwar Guatemala », dans Blom Hansen T. et Stepputat F. (eds.), States of Imagination. Ethnographic Exploration of the Postcolonial State. London/Durham, Duke University Press, 2001, p. 203-220.

Sonnleitner, Willibald, « Aux marges de la démocratie : 22 ans de processus électoraux au Guatemala », Problèmes d'Amérique latine, n73, 2009, p. 37-72.

Thiesse, Anne-Marie, La création des identités nationales. Europe, XVIIIe, XXe siècle, Paris, Points, 2010.

Vanthuyne, Karine, La présence d'un passé de violences. Mémoires et identités autochtones dans le Guatemala de l'après-génocide, Laval, Presses de l'Université de Laval, 2014.

\section{NOTES}

1. Déclaration publiée par le ministère de la Culture et des Sports. «Unis dans la célébration [et] la sauvegarde de notre identité nationale, reconnaissant notre diversité, cherchant la réconciliation et l'harmonie sociale ".

2. Ces accords ont mis fin au conflit armé (1960-1996) qui opposait des mouvements de guérilla marxistes aux gouvernements dictatoriaux successifs, après le pic de violence des années 1970-1980.

3. En mai 2021, plusieurs députés ont déposé une proposition de loi dite de « consolidation de la paix et réconciliation » qui visait à appliquer l'amnistie négociée lors des Accords de paix à l'ensemble des délits commis durant le conflit, y compris les délits de crimes contre l'humanité, qui restent condamnables, argumentant que des délits tels que le génocide n'existaient pas dans le droit guatémaltèque.

4. L'accès à l'eau, les routes ou les bâtiments scolaires sont fréquemment construits de manière collective à l'échelle du municipe.

5. Notamment l'affaire de la línea en 2015, impliquant le président de l'époque, Jimmy Morales.

6. Maya, Xincas et même "métis" étant alors rassemblés par une expérience commune de l'oppression exercée par le gouvernement central. 


\section{AUTEUR}

\section{CLARA DUTERME}

Clara Duterme est anthropologue et membre du laboratoire Ades (Aix Marseille Université, AMU). Ses recherches portent sur les enjeux identitaires et mémoriels au Guatemala. Dans le cadre du programme de recherche TRANSFUNÉRAIRE, elle étudie en particulier les pratiques d'exhumations et de ré-inhumation des victimes du conflit. Elle a récemment publié l'article « La violencia en la tierra. Una mirada a las relaciones de poder en Guatemala desde las fosas de exhumación vacías » (NuevoMundo MundosNuevos, Questions du temps présent, 2021).

clara.duterme@univ-amu.fr 